\title{
Interventores y comunidades vulnerables: una propuesta de formación para universitarios
}

\author{
Dulce Nayeli Cohetero-Hernández \\ Licenciada en Música. Magíster en Innovación Educativa \\ Secretaría de Educación Pública. Chihuahua, México \\ https:// orcid.org/0000-0001-5085-0140 • $\underline{\text { silce207@hotmail.com }}$
}

\author{
Fidel González-Quiñones \\ Licenciado en Ciencias de la Información. Magíster en Mercadotecnia \\ Universidad Autónoma de Chihuahua. Chihuahua, México \\ https:// orcid.org/0000-0002-8404-0098 • fgonzalez@uach.mx
}

Resumen

La presente investigación se centra en la propuesta y validación de un programa de formación de interventores preparados para incidir en grupos vulnerables. La formulación se realizó con fuentes bibliográficas y trabajo colaborativo con expertos en esta área en los Estados de Veracruz y Chihuahua; la validación se hizo mediante consulta a expertos en el Estado de Chihuahua, analizando la pertinencia de cada constructo del programa con la técnica Delphi. Los resultados obtenidos denotan la importancia de capacitar a los interventores para que conciban proyectos y visualicen a las poblaciones a intervenir desde sus posibilidades de desarrollo y no desde sus vulnerabilidades, las cuales deben entenderse como algo relativo y no inherente. La propuesta se basa en el trabajo colaborativo, tanto en la formación de interventores como en el diseño, aplicación y evaluación de las intervenciones a realizar, buscando la integración de la población objetivo a actividades tradicionalmente pensadas para coordinadores o interventores de los programas. Se resalta la importancia de que el interventor sea formado en el conocimiento y respeto del entorno y tradiciones de las comunidades a intervenir, para que su meta de desarrollo propuesta sea compatible con los valores y cosmogonía de dicha comunidad. Por último, se encontró la necesidad de ahondar y abogar por la necesidad del uso de indicadores, matrices FODA y presupuestos en las intervenciones sociales.

Palabras clave: Intervención social; Trabajo colaborativo; Vulnerabilidad social; Educación.

Recibido: 24/05/2019 | Aprobado: 15/10/2019

(i)(2) Esta obra está bajo una Licencia Creative Commons Atribución-NoComercial-CompartirIgual 4.0 Internacional.

Financiación o proveniencia del artículo: Artículo derivado del proyecto de la Maestría en Innovación Educativa con línea de investigación Procesos educativos innovadores inscrito en Programa Nacional de Posgrados de Calidad de la Universidad Autónoma de Chihuahua avalado por Conacyt y llevado acabo entre los años 2016 y 2018.

¿Cómo citar este artículo? / How to quote this article?

Cohetero-Hernández, D.N., y González-Quiñones, F. (2020). Interventores y comunidades vulnerables: una propuesta de formación para universitarios. Prospectiva. Revista de Trabajo Social e intervención social, (29), 131-150. doi: 10.25100/prts.v0i29.7938. 
Cohetero-Hernández y González-Quiñones

\title{
Intervenors and Vulnerable Communities: a Training Proposal for University Students
}

\begin{abstract}
This investigation focuses on the proposal and validation of a training program for intervenors prepared to intervene in vulnerable groups. The formulation was constructed with bibliographic sources and with collaborative work with experts in this field in the States of Veracruz and Chihuahua; the validation was done by consulting experts in the State of Chihuahua, analyzing the relevance of each construct of the program with the Delphi technique. The results showed the importance of training intervenors to conceive projects and visualize the target populations focusing on development possibilities rather than vulnerabilities, which should be understood as relative and not inherent. The proposal is based on collaborative work, both in the training of intervenors and in the design, application and evaluation of the interventions to be executed, seeking the integration of the target population in activities traditionally designed for program coordinators or intervenors. The importance of the auditor being trained in the knowledge and respect of the environment and traditions of the communities to be intervened is highlighted, so that their proposed development goal is compatible with the values and cosmogony of said community. Finally, the study discovered the need to advocate for the use of indicators, SWOT analyses and budgets in social interventions.
\end{abstract}

Keywords: Social intervention; Collaborative work; Social vulnerability; Education.

Sumario: 1. Introducción, 2. Metodología, 3. Revisión de la literatura, 3.1 Intervenciones Educativas, 3.2 Intervención Social, 3.3 Percepción del interventor sobre los participantes vulnerables, 3.4 Grupos de desarrollo autogestivos, 4. Contexto de la Investigación, 5. Hallazgos, 5.1 Trabajo Colaborativo con Expertos, 5.2 Validación del programa de formación propuesto, 6. Conclusiones, 7. Referencias bibliográficas. 


\section{Introducción}

Un modelo de institución que en cada región es de los principales ejes culturales y promotores de desarrollo es la Universidad; sin embargo, en la mayoría de los casos los estudiantes buscan realizar su servicio social al cobijo de un programa, proyecto o institución de servicio social ya establecido, que junto a muchos beneficios y facilidades trae consigo exigencias en metodología, límites en tiempos y objetivos preestablecidos a cumplir que usualmente vienen de una tradición paternalista/asistencialista. A veces los lineamientos y requisitos para pedir recursos y favorecer una intervención de origen civil tienen más que ver con el cumplimiento de requisitos presupuestales que con buscar que las personas en estado de vulnerabilidad superen tal situación. Así, los jóvenes que se aventuran a intervenir en grupos vulnerables se enfrentan a la doble dificultad de plantear un plan de intervención desde la experiencia y conocimiento en su área particular; y aprender sobre la marcha a realizar una intervención cuya naturaleza suele estar entre lo educativo y la asistencia social.

No se puede comprender el estado actual de los programas de mejora social sin conocer la historia de las políticas económicas por las que ha pasado el México moderno. Comenzando por El Modelo de Crecimiento Económico, establecido desde los 40’s señalado por Salas (2009), cuyos principios básicos fueron la sustitución de importaciones, el desarrollo hacia adentro y la economía mixta donde, según Ramos (2000), era el Estado quien fungía como agente regulador y era el responsable de impulsar el desarrollo social; sin embargo, desde 1982 se aplica un nuevo sistema: el Neoliberalismo, que trata de responder a las tendencias propias de la globalización insertando ideas como la aldea global y la capacidad de consumo de productos y servicios: "como nunca antes, impone estándares a millones de personas en un flujo inimaginable de cauces y significados" (Castells y Borja, 2002, p. 418). Bajo este esquema, el mercado substituye al Estado en la función de regulador del desarrollo social.

Descrito por Laval y Dardot (2017) como: "un conjunto de relaciones de poder mediante las cuales las sociedades y sus instituciones, así como también la naturaleza y las subjetividades, son sometidas a la ley de la acumulación del capital financiero" (p. 87), este nuevo modelo económico también trae un nuevo sistema de valores donde el poder adquisitivo es la medida de realización personal y bienestar trayendo como consecuencia que en algunos círculos sociales se llega a extremos, donde el enriquecimiento rápido a costa de los demás es visto como signo de inteligencia y prestigio.

Otro elemento que ha permeado la cotidianeidad mexicana llegando hasta el ambiente escolar es el narcotráfico, con la consecuente ola de violencia que esta actividad ha desencadenado en las últimas décadas. Si bien en muchas regiones de México existe un 
proceso de adaptación y de lucha por rescatar el espacio público, es el Estado al que se dedica este trabajo, Chihuahua, uno de los que más ha sufrido este fenómeno social, económico y político del narcotráfico. En el año 2010 el estado de Chihuahua alcanzó el primer lugar de violencia extrema e inseguridad en México y el mundo, según el Instituto Nacional de Estadística y Geografía (2016). En ese año se registró el mayor número de homicidios desde 1990, llegando a la cifra de 6,407, convirtiendo en pocos meses sus calles en escenarios de violencia extrema. La misma fuente estableció que el $63.40 \%$ de la población de Chihuahua percibe su entorno como inseguro, lo cual significa que más de la mitad de la población de este estado se siente insegura en el transporte público, bancos, cajeros, calles que usa habitualmente, mercados, carreteras y parques recreativos. Por ello este trabajo de investigación buscó hacer una aportación para la solución de estas problemáticas, mediante la formación de estudiantes universitarios como interventores capacitados para el trabajo con grupos vulnerables.

Los objetivos de este estudio se desglosan en un objetivo general y tres específicos. El objetivo general es determinar las características de un programa de formación para estudiantes universitarios, que les permita realizar actividades como interventores en grupos vulnerables en la ciudad de Chihuahua. Los objetivos específicos son: a) determinar qué contenidos debe contemplar un programa de formación para estudiantes universitarios como interventores en grupos vulnerables; b) determinar las características de validez en cuanto a pertinencia del programa propuesto; c) determinar los valores y actitudes que debe desarrollar un programa de formación para estudiantes universitarios como interventores en grupos vulnerables.

Teniendo en cuenta las situaciones de vulnerabilidad en las que viven miles de personas en la ciudad de Chihuahua, es necesario tomar iniciativas desde una perspectiva holística, que busquen empoderar a los beneficiarios de los programas de asistencia para que se vuelvan agentes de cambio de su entorno. Los mediadores para este objetivo en el presente estudio fueron los estudiantes de la Universidad Autónoma de Chihuahua, puesto que la Universidad es una institución que tiene como función la transición y generación de nuevo conocimiento, además del respaldo moral que brinda el ser referido por este tipo de instituciones. La importancia que se le da en esta propuesta a buscar la capacitación de los interventores para promover la formación de grupos autogestivos, tiene que ver con lo expuesto por autores como Castel (2004) y Montero (2014), quienes recalcan la trascendencia de la formación de capital humano.

Por ello fue necesario recopilar información sobre el contexto de las personas en situación de vulnerabilidad en el Estado de Chihuahua y de intervenciones o estudios previos en dicho Estado para adaptar un programa de formación que les permita a los estudiantes de nivel universitario aplicar intervenciones sociales y programas en comunidades vulnerables, pudiendo estos ser parte de su servicio social, sus actividades extracurriculares o como una forma de ejercer su carrera al egresar de la universidad.

Prospectiva. Revista de Trabajo Social e intervención social • No. $29 \bullet$ ene.-jun. 2020 • pp. 131-150 e-ISSN: 2389-993X • Doi: 10.25100/prts.v0i29.7938 Universidad del Valle - Cali, Colombia 


\section{Metodología}

La naturaleza de la investigación fue cualitativa, aplicada, no experimental transeccional descriptiva.

El interés principal de este estudio es la fundamentación tanto en la teoría como en los saberes de los colaboradores de una experiencia de formación capaz de emancipar, criticar e identificar el potencial para el cambio en grupos vulnerables, o como prefieren ser llamados: grupos con oportunidades de desarrollo. Los objetivos anteriores pretenden ser alcanzados incidiendo en la transformación de estos grupos mediante una perspectiva Glocal, como lo propone Touriñán (2011) y holística; con el propósito de plantear una hipótesis de trabajo en un contexto y tiempo dado de manera inductiva para la transformación de la realidad. La metodología que se consideró más adecuada para responder tanto a los objetivos del estudio como al paradigma y enfoque seleccionados fue la investigación colaborativa.

El procedimiento usado para la colecta de la información y su procesamiento para derivar resultados fue el etnográfico, mediante las técnicas de observación, entrevistas y análisis cualitativo. El modo de realización fue de campo con apoyo bibliográfico. Para la validación se recurrió a expertos mediante un instrumento basado en la técnica Delphi, para analizar la pertinencia de cada constructo del programa de formación propuesto.

\section{Delimitación de la investigación}

La investigación se realizó de agosto del 2017 a octubre del 2018, la cual fue la duración del programa de maestría que sirvió de marco para este trabajo, más el tiempo de recolección de las retroalimentaciones de los expertos para la validación del programa de formación propuesto.

La demarcación geográfica de las acciones de la investigación fue dentro de los estados de Chihuahua (observación, entrevistas, análisis cualitativo y validación por expertos) y en menor medida en Veracruz (observación, mesa de trabajo y entrevistas). Esto último, durante una estancia de investigación realizada de febrero a marzo del 2018.

\section{Población de interés}

Para este estudio se utilizó una selección no probabilística por conveniencia. El trabajo colaborativo se realizó en el marco del estado de Chihuahua y el de Veracruz por la posibilidad de tener acceso a expertos de los tres niveles en estos Estados y poder observar directamente el proceso de la intervención.

Este estudio reunió a 17 expertos en diferentes actividades de las intervenciones, cuya distribución por Estado de la República y categoría de función se muestra en la Tabla 1, de 
Cohetero-Hernández y González-Quiñones

los cuales $52.94 \%$ fueron hombres y $47.05 \%$ mujeres; sobre los rangos de edad, el $23.52 \%$ de los participantes tenían edades entre los 20 a 30 años, $41.17 \%$ en edades de 31 a 40 años y $35.29 \%$ tenía 41 años o más al momento de su participación en este estudio. Sobre la zona por actividad económica en la que se desarrollaban los participantes, el 35.30\% vivía en una zona rural y el $64.70 \%$ en zona urbana. Los expertos que participaron en la reformulación colaborativa del programa de formación se dividieron en tres categorías, dependiendo del rol en el que tenían más tiempo de experiencia. Esta decisión se tomó puesto que en los grupos autogestivos, los beneficiarios de los proyectos suelen pasar a tomar otros roles conforme el proyecto se va consolidando y es común que los interventores y diseñadores de proyectos realicen actividades pensadas para los participantes; estas categorías fueron: diseño de intervenciones $(n=4)$, implementación de intervenciones o interventor $(n=3)$ y participación en intervenciones $(n=6)$. En cuanto a la validación del programa, se contó con el apoyo de 4 expertos, quienes contaban con experiencia mayor a dos años en investigación educativa, en el Estado de Chihuahua, y actividades relacionadas con la formación de formadores, de los cuales una pertenecía al sexo femenino y tres al masculino.

Tabla 1. Caracterización de los participantes en el estudio.

\begin{tabular}{lcc}
\hline Actividad & Chihuahua & Veracruz \\
\hline Diseño de intervenciones & 2 & 2 \\
Interventor & 2 & 1 \\
Participante & 2 & 4 \\
Experto validador & 4 & 0 \\
\hline Total: & 10 & 7
\end{tabular}

Fuente: Elaboración propia

\section{Operacionalización de Variables}

Las variables a medir en este estudio corresponden a las usadas para validar la propuesta del programa de formación, como Variable Dependiente (Y) se tiene la Validez de pertinencia y como variable independiente $(X)$, está la Validez del programa de intervención.

\section{Instrumentos}

Los instrumentos que se usaron en este estudio fueron la Carta de presentación y validación del programa de formación y la Guía para entrevistas semiestructuradas con expertos en intervenciones. La primera para valorar la validez de pertinencia en los constructos del programa de formación; este instrumento se basa en la técnica Delphi y consta de 17 ítems valorados numéricamente del uno al cuatro, de los cuales el uno refleja un nivel de pertinencia nula, el dos una pertinencia baja, el tres una pertinencia alta y el cuatro una 
pertinencia óptima. El mismo contaba con una sección por cada constructo para que los expertos vertieran sus comentarios o sugerencias de manera abierta.

Por su parte, la Guía para entrevistas semiestructuradas con expertos en intervenciones, basada en el guion de entrevista para representantes de la OSC en Tlaxcala, constaba de 17 preguntas abiertas.

\section{Procedimiento de la investigación}

La investigación se realizó en las siguientes cuatro etapas:

a. Diseño del programa de formación, donde teóricamente se delimitan las características de un interventor en grupos vulnerables.

b. Acercamiento para enriquecer el programa a expertos en tres niveles: planeación, implementación y participación en intervenciones a grupos vulnerables. En todos los casos, presenciando de primera mano sus actividades dentro de las comunidades.

c. Elaboración de propuesta de intervención integrando los conocimientos y saberes de la investigación colaborativa

d. Validación con expertos: a través de la técnica Delphi se analizó la validez de pertinencia de cada constructo del programa de formación, se realizó una tabla de contingencia y un análisis de contenido de las aportaciones de los expertos.

\section{Revisión de la literatura}

\subsection{Intervenciones Educativas}

La palabra intervenir significa estar entre dos momentos diferentes, un antes y un después, tomar partido en esta transición. Según Remedí (2004), la intervención está entre lo que está instituido y un proceso que es instituyente. Citando a Touriñán (2011, p. 283): "La intervención educativa es la acción intencional para la realización de acciones que conducen al logro del desarrollo integral del educando". Las intervenciones educativas deben trabajar sobre procesos emergentes. Toda intervención se da dentro de una comunidad definida; en el caso de la Institución Educativa (IE), la comunidad es la escuela y su contexto, y ambos deben ser tomados en cuenta, pues cada uno querrá que la intervención educativa tenga ciertas características que respondan a sus necesidades, gustos e historia personal. También se deben de contemplar, según Remedí (2004), los "haceres" de la comunidad, las prácticas instituidas de los docentes que tienen que ver con la cultura institucional. 
Una intervención es un programa específico que tiene el objeto de ayudar a un educando en un área de necesidad específica. Sus características según Lee (2015) y Touriñán (2011) son:

a. Tienen una intención definida y limitada.

b. Son específicas y con una duración delimitada.

c. Tiene carácter teleológico (analiza: ¿para qué educar?, ¿cuáles son los fines de la educación?).

d. Se realiza mediante procesos de autoeducación y heteroeducación.

e. Respeta al educando como agente para no incurrir en coacción.

A pesar de ser formales, las intervenciones educativas pueden tener flexibilidad para adaptarse a las necesidades particulares de cada sujeto o grupo de intervención.

Según Remedí (2004), no todo mundo es apto para realizar intervenciones y se requiere contar con varias cualidades; se debe aceptar que toda intervención va a ser un acto de negociación de significados, pues no se puede llegar a imponer significados a los educandos. Tampoco va a resultar exactamente como se ha planeado, pues los educandos son sujetos y no meros objetos en ella. Se debe tener la suficiencia técnica necesaria para impartir el contenido previsto, que se manifiesta al reconocer qué destrezas, hábitos, aptitudes y actitudes de los diversos dominios que señalan las taxonomías se potencian al momento de trabajar de cierta forma cada tema (Touriñán, 2011). Para esta misma fuente, toda intervención debe estar a la altura de su tiempo, debe lograr la creación de hábitos de desarrollo intelectual en el educando, desarrollo afectivo, de auto-construcción y construcción del proyecto de vida personal desde sus áreas de experiencia. Se debe educar "con" el área cultural y no "en" esta; es decir, hay que concebir a la educación como: "una actividad orientada al uso y construcción de uno mismo, para asumir como parte de nuestra educación el carácter axiológico, personal, integral y patrimonial, propio de toda educación, y el sentido intercultural, glocal, permanente, general y profesional de una educación a la altura de los tiempos" (Touriñán, 2011, p. 302). Entendiendo lo glocal como una perspectiva de conocer, adquirir y valorar tanto lo local como lo global.

\subsection{Intervención Social}

La intervención social (I.S.) es una unidad desde las intenciones hasta las acciones y relaciones con la población a intervenir, siempre focalizando en las planeaciones el qué acciones tomamos, cómo y para qué (Habermas, 1997; Herrera y Castón, 2003).

Según varios autores, las mejores metodologías para definir una línea de acción al momento de buscar un cambio actitudinal son las que permiten comprender al sujeto en relación con sus acciones y contextos sociales, como son: las metodologías participativas, las 
Cohetero-Hernández y González-Quiñones

de la planificación radical, metodología comunicativa crítica, aprendizaje en acción o el acompañamiento social (Arenas, 2013; Castel, 2004; Friedmann, 2001; Gómez, Latorre, Sánchez y Flecha, 2006; Habermas, 1987 y 1997; Rodríguez, 1994; Supiot, 1999).

La sugerencia más común es iniciar con grupos de discusión con pretensiones comprensivas y realizar a ellos una meta-lectura para hallar indicaciones sobre qué acción de intervención es la más apropiada (Arenas, 2013).

\subsection{Percepción del interventor sobre los participantes vulnerables}

Durante las últimas décadas se han realizado diversos estudios con el objetivo de cambiar de actitud con respecto a una población vulnerable (Aguado y Alcedo, 1999; Byrd \& Elliott, 1988; Donaldson, 1987; Elliott \& Byrd, 1982). La investigación ha puesto de relieve que las actitudes hacia las personas con algún tipo de vulnerabilidad no han sido ni son predominantemente positivas. Esta situación plantea la conveniencia y urgencia de poner en marcha campañas de sensibilización con el objetivo de promover cambios en tales actitudes. Las principales metodologías para lograr el cambio de actitudes fueron la información directa e indirecta sobre el grupo vulnerable, además del contacto con estos grupos en sesiones interactivas con discusión guiada (Aguado y Alcedo, 1999; Byrd \& Elliot, 1988; Donaldson, 1987; Elliot \& Byrd, 1982). En todos ellos se ha destacado la factibilidad de lograr un cambio de actitud de unas negativas a otras consideradas como positivas, "a partir de la manipulación de uno de los componentes actitudinales" (Aguado, Flórez y Alcedo, 2004). Por el contrario, varios estudios reportaron que las actitudes positivas logradas van decayendo de modo estadísticamente significativo a lo largo de medidas de seguimiento, aunque el efecto del tratamiento no llega a desaparecer totalmente (Verdugo, Jenaro y Arias, 1995; Wright, 1984; Yuker, 1988), aunque proponen que estos cambios se deben a que sus grupos experimentales eran más pequeños que sus grupos de control, a los efectos residuales de usar una misma medición repetidamente, a la regresión estadística y a la maduración de los participantes.

La intervención comunitaria pretende capacitar y fortalecer a la comunidad para que sea ejecutora y gestora de su propia transformación y la de su entorno. Mori (2008), propuso el uso de una metodología cualitativa y participativa para lograr incidir en la psicología comunitaria, además estableció que la intervención se debía resumir en las siguientes fases: el diagnóstico de las necesidades de la comunidad, el diseño y planificación de la intervención, evaluación inicial de la planeación previa a la planificación, evaluación final y la diseminación o exposición de los resultados obtenidos.

Finalmente, algunos investigadores han resaltado la utilidad del análisis FODA como una herramienta para visualizar y ordenar información relevante del modelo de 
Cohetero-Hernández y González-Quiñones

intervención antes de ejecutarla (Idáñez, Ander-Egg y Richmond, 2001; Matzumura, Ayala, Aguilar, y Gutiérrez, 2008). Este análisis consiste en poner en un diagrama las circunstancias internas del grupo o comunidad, tanto positivas (fortalezas), como negativas (debilidades); así como visualizar las circunstancias externas positivas (oportunidades) y negativas (amenazas), siendo la base para plantear estrategias para aumentar las posibilidades de éxito de las I.S.

\subsection{Grupos de desarrollo autogestivos}

La autogestión como discurso político, se origina, según diversos autores (Arvon, 1982; Mendizábal y Errasti, 2008; Rosanvallon, 1979) en el pensamiento libertario, es decir, en la militancia que aboga por la anarquía. Al contrario, otras fuentes (Arvon, 1982; Mintz, 2006; Rosanvallon, 1979) han recogido diversas acciones autogestivas en diversos lugares del mundo como el pensamiento socialista, consejos obreros en Italia y Alemania y otras formas de organización tradicionales, por mencionar algunos ejemplos. Según Gómez (2013), este concepto de autogestión apareció en la década de los 70’s. Tomando en cuenta su concepción desde el ámbito libertario, la autogestión se caracteriza por:

a. Romper la lógica de las clases sociales

b. Implicación de los individuos en las decisiones del grupo social

c. No existencia de agentes gubernamentales que generen estructuras jerárquicas involucrados en el diseño de proyectos o toma de decisiones

Difiriendo con este último punto, según Dahl-Ostergaard, Moore, Ramírez, Wenner y Bonde (2003), es posible la implicación de prestadores de servicios, autoridades locales, Organizaciones no Gubernamentales, y demás agentes externos en el planteamiento de un proyecto autogestivo, cuando éste busca cumplir metas de desarrollo con un origen ajeno pero compatible con los valores de la comunidad a intervenir.

Puede verse a la necesidad de este sistema de autoprotección-autogestión como una reacción al paso del modelo económico de bienestar o proteccionista, donde Estado intervenía activamente al distribuir la riqueza, al modelo neoliberal, donde la distribución queda a cargo del mercado; sin embargo, según Gómez (2013), después de más de 40 años de este modelo, quedaron secuelas negativas en las poblaciones: “desempleo, la falta de oportunidades para insertarse en el mercado de forma competitiva, el acaparamiento de mercados por parte de monopolios, la pérdida de capacidad adquisitiva y la pérdida de derechos de toda índole" (p. 2). Este mismo autor relaciona los efectos negativos de este sistema económico con el aumento de la violencia y reducción del nivel de vida en los países en desarrollo. Tal como lo señalan diversos autores (Charkiewicz, 2005; Ezcurra, 1998; Hale, 2002; Harvey, 2005; Ortiz, 2010) el ciudadano ha perdido tal estatus frente al Estado y se ha convertido en un consumidor, por lo cual el Estado se administra como empresa privada 
recortando gastos en lo social, la cual debería ser su principal función; además de que éste último "gasto", según Duhau (2002), lo focaliza en poblaciones que considera vulnerables bajo sus propios términos. Diversos autores (Gómez, 2013; Ortiz, 2010; Paley, 2001) coinciden en que este vacío en asistencia social debe ser llenado por los mismos ciudadanos, teniendo un rol activo para la resolución de sus problemas y búsqueda de opciones para su desarrollo, aunque también los autores reconocen a la tradición paternalista en México como un obstáculo para que los ciudadanos lleguen a ejercer plenamente estas nuevas funciones (Gómez, 2013; Ortiz, 2010; Rodríguez y Torres, 1994), puesto que ciudadanos, políticos y funcionarios de gobierno siguen aplicando medidas de corte paternalista. Esta "transferencia de poder" connota para Gómez (2013) la concepción de estas comunidades como carentes de poder desde la perspectiva de los interventores, lo que es incongruente con el sentido libertario de los grupos autogestivos, que no conciben la necesidad previa de empoderamiento.

Aun cuando pareciera restarse importancia en estos proyectos a las comunidades, dado que tanto la iniciativa como los recursos provienen en su mayoría del exterior "Es en los espacios locales donde adquiere forma, se concreta y potencializa el proceso de desarrollo" (Enríquez, 2011, p. 189). Es en lo local donde se acentúan las fallas y los aciertos en la toma de decisiones en políticas públicas a nivel nacional y es en lo local donde el plan se encuentra con la realidad y se pone a prueba en su factibilidad, pertinencia y eficacia.

\section{Contexto de la investigación}

\section{Contexto en México}

En México se han creado diversas instituciones e instituido normas para guiar y regular los esfuerzos nacionales encaminados al desarrollo, por ejemplo: la Secretaría de Desarrollo Social (SEDESOL) en 1992, la creación del Sistema de Protección Social en Salud en 2005, la publicación de la Ley General de Desarrollo Social en 2004, y la creación del Consejo Nacional de Evaluación de la Política de Desarrollo Social (CONEVAL) en 2005.

Según el ANUIES (2016), en México hay 2,107 instituciones de educación superior (IES), de las cuales 699 son públicas. Obedeciendo a su coordinación, éstas se clasifican en ocho grandes grupos: 66 universidades públicas, 213 instituciones tecnológicas, 27 Centros CONACYT, 78 instituciones públicas que dependen de otras Secretarías de Estado entre ellas, la Escuela Nacional de Antropología e Historia, el Instituto Nacional de Bellas Artes, las IES agropecuarias, las de las fuerzas armadas y las del sector salud. Existen 60 universidades tecnológicas más la Universidad Autónoma de la Ciudad de México; las universidades interculturales, de reciente creación; 1,408 instituciones particulares de educación superior, en las cuales se encuentran matriculados un total de 3,915,975 estudiantes, de los cuales 1,976,811 son hombres y 1,939,160 son mujeres. 


\section{Dimensión comunitaria en Chihuahua}

El arraigo de los jóvenes a su colonia o barrio se puede considerar alto según el Instituto Nacional de Estadística y Geografía y Secretaría de Gobernación (2014), pues el $47.50 \%$ de los encuestados no se mudaría aún si tuviera los medios necesarios; de los encuestados que sí estarían dispuestos a mudarse, el 53.80\% lo haría a otra colonia o barrio y el porcentaje restante saldría de su barrio a otra ciudad, estado o país.

La organización de vecinos para objetivos comunes no es un evento que se perciba con mucha frecuencia, pues sólo el $24.20 \%$ de los encuestados ha observado estas reuniones para fines religiosos; el $18.40 \%$ para organizar fiestas; sólo el $16.70 \%$ de las reuniones para tratar de solucionar problemas de servicios públicos y $14.80 \%$ para organizar la seguridad de la colonia. Por último, vemos que sólo un $10.80 \%$ se reúnen para convivir de manera casual.

Sólo el 15.90\% de los jóvenes entre 12 a 29 años participa en actividades o programas en su colonia o barrio. De estos, el $59.10 \%$ lo hace en un grupo deportivo y sólo un $8.70 \%$ en un grupo artístico. Las principales causas por las que no se participa en actividades o programas son: la falta de tiempo, inexistencia de actividades o programas y en menor medida la falta de interés. Los adultos cuestionados en este sentido expresaron que no se relacionan en estas actividades por falta de tiempo (38.80\%). Por otro lado, el $86.60 \%$ de los que si participan en actividades percibe que su participación influye en el desarrollo de su colonia o barrio.

Dentro de las conductas delictivas ante las cuales los vecinos son más propensos a actuar en conjunto son las peleas entre pandillas con un $50.60 \%$ de acción conjunta y los robos con un $39.90 \%$.

\section{Hallazgos}

\subsection{Trabajo Colaborativo con Expertos}

Durante el debate y la mesa de trabajo se presentaron los resultados de la revisión de literatura sobre intervenciones socioeducativas, factores de vulnerabilidad de la población, prácticas de intervención en grupos vulnerables en el contexto de Chihuahua y las dificultades en relación a las diversas necesidades y características de los destinatarios de dichos grupos; también se abordaron temáticas como la colonización mediante la educación y los problemas éticos que enfrentan los interventores. El análisis de las percepciones de los participantes dio como resultado la creación de tres dimensiones: Vulnerabilidad, Construcción de comunidad, y Autopercepción de los interventores 
Cohetero-Hernández y González-Quiñones

a. Vulnerabilidad: Una importante reflexión encontrada tanto en los interventores como en los participantes de ambos estados fue que la vulnerabilidad no es un rasgo inherente de una etnia, religión o género; es percibida como efecto del entorno en un $84.60 \%$ de los casos (factores políticos, culturales y económicos). El 76.90\% de los entrevistados percibe a las I.S. como un factor de disminución de la vulnerabilidad. Uno de los participantes expresó: "estar aquí, viniendo, parece poco, pero abre la mente, te das cuenta de un montón de cosas".

Algo a resaltar fue la petición de algunos participantes de que su comunidad no fuese denominada "grupos vulnerables"; aunque esto responde al análisis de la bibliografía, sugieren que eso ya impone un prejuicio en la mente de los interventores al momento de interactuar con la comunidad.

La consideración de la inseguridad en el diseño de la I.S. se realizó en mayor medida en Chihuahua, aunque se hicieron adaptaciones al respecto por los interventores en todos los casos.

El 53.80\% de los encuestados (en su mayoría participantes e interventores), se perciben en una situación de abandono por las instituciones de gobierno en general.

b. Construcción de Comunidad: Aunque todos los diseñadores de I.S. expresaron su preocupación por construir comunidad, de los 4 diseños analizados, sólo el $75,00 \%$ hace explícito este objetivo. El $84.60 \%$ de los entrevistados percibe a la I.S. como herramienta para el rescate del tejido social, el $76.90 \%$ como herramienta para el fomento de valores, y el $92.30 \%$ como instrumento para el desarrollo individual.

Se encontraron percepciones tanto negativas como positivas de los participantes sobre la forma en la que los investigadores se acercan a las comunidades, siendo las más comunes las expresadas en la Figura 1, donde se muestran los registros de ambos estados por separado y un cálculo en porcentaje de la población total de participantes. 
Cohetero-Hernández y González-Quiñones

Figura 1. Percepciones de los participantes sobre los interventores

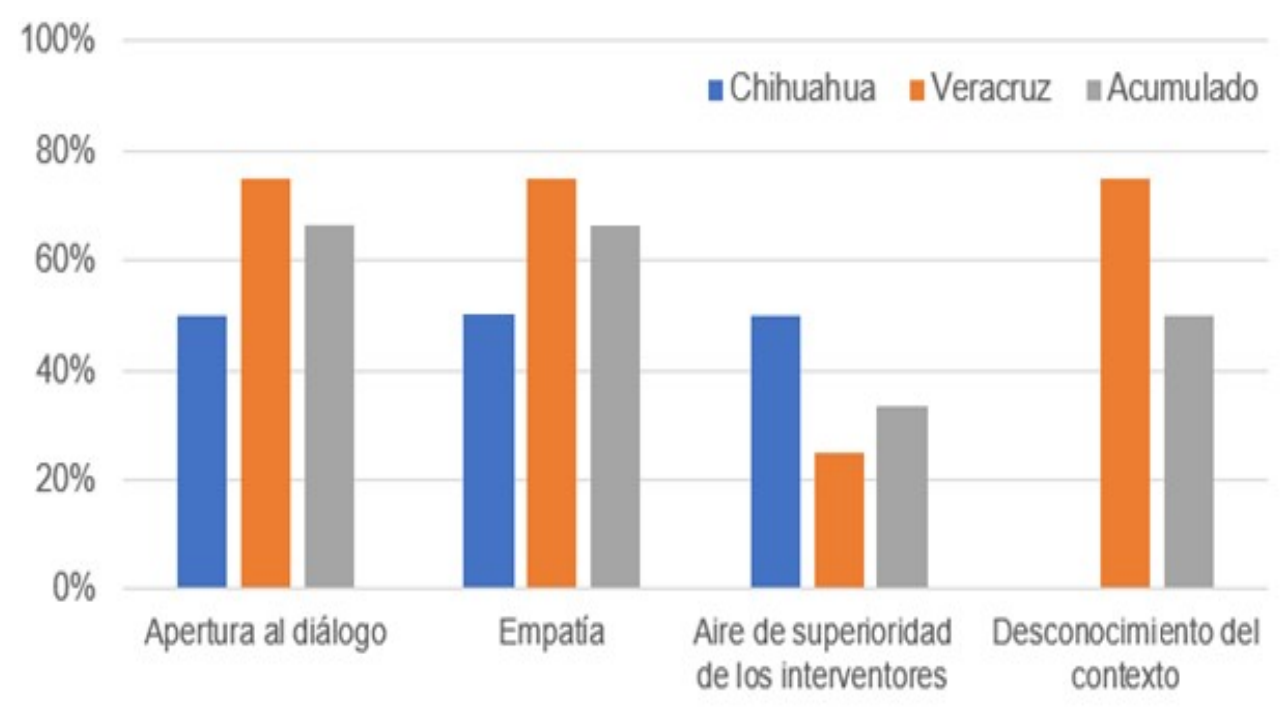

Fuente: elaboración propia

c. Autopercepción de los Interventores: De 3 interventores entrevistados, 2 perciben que les falta conocimiento en cuanto a procesos o metodologías didácticas. El interventor de Veracruz expresó no haber sido consiente del desconocimiento de la realidad a intervenir hasta el momento de la implementación de la I.S.; ninguno de ellos recibió capacitación especial como interventor. En cuanto a los diseñadores de las intervenciones, los dos casos de Chihuahua habían recibido formación especializada para realizar intervenciones, mientras sus contrapartes de Veracruz usaban su experiencia y materias afines de maestría o doctorado para dirigir sus acciones.

\subsection{Validación del programa de formación propuesto}

Para realizar la validación del programa de formación propuesto, se buscó el apoyo de expertos en el área de la investigación educativa y de campo sobre el Estado de Chihuahua. En la Tabla 2, se muestran los temas de dicho programa que obtuvieron incidencias mayores a dos en las entrevistas con expertos. Primero se expondrán los resultados, para concluir con los comentarios personales y conclusiones sobre este proceso. 
Tabla 2. Contingencia de las aportaciones de los expertos por tema del programa de formación propuesto.

\begin{tabular}{lc}
\hline Tema & Incidencia \\
\hline Vulnerabilidad & 4 \\
\hline Del asistencialismo a la autogestión & 3 \\
Importancia de las intervenciones & 2 \\
Selección de población & 2 \\
Identificación de necesidades culturales y sociales & 2 \\
Creación de indicadores & 2 \\
Evaluación de la intervención & 2 \\
\hline
\end{tabular}

Fuente: elaboración propia

Vulnerabilidad: De los cuatro expertos, uno señaló que debe explicarse el origen de dicha palabra como "la cualidad que tiene alguien para poder ser herido" y hacer más explícito el género como un factor importante para ser vulnerable. Es en este último punto donde hubo consenso entre los expertos. Tres de los cuatro expertos coincidieron en tomar la visión de este tema desde la discapacidad, equidad de género, pobreza extrema y etnias. Otro experto señaló la importancia de no ver a la persona desde su vulnerabilidad, si no desde sus posibilidades de desarrollo y participación.

Del asistencialismo a la autogestión: Un experto comentó que se ha demostrado que los resultados de las intervenciones en comunidades son mejores si la población objetivo es la que define si se efectúa la intervención y cómo llevarla a cabo; otro mostró interés en la capacidad de convertir a las poblaciones vulnerables en comunidades emprendedoras. Un experto recomendó trabajar este tema después de los grupos vulnerables en Chihuahua.

Importancia de las intervenciones: Un experto recalcó que debe mencionarse en el programa de formación que las decisiones se toman de forma colegiada en el trascurso del mismo. Dentro de este tema, otro experto enfatizó la importancia de eliminar las barreras que impiden el aprendizaje o participación social.

Selección de población: Un experto recomendó que al momento de formar los grupos a los que se aplicaría este programa de formación, se cuide que estos tengan una distribución de sexo de 50,00\% y 50,00\%, puesto que la percepción de la vulnerabilidad es diferente para cada género. Sobre la población a intervenir como parte del trabajo de los participantes de este curso de formación, un experto comentó que no debe segregarse a las personas vulnerables, la comunidad debe convivir y aprender junta.

Identificación de necesidades culturales y sociales: Un experto no entendió el contenido de este tema. Otro felicitó por su inclusión al programa siempre y cuando se realice en comunidad. 
Cohetero-Hernández y González-Quiñones

Creación de indicadores: Un experto no entendió la utilidad de este tema para el programa de formación. Otro felicitó por su inclusión y comentó la importancia de medir el impacto de proyectos sociales de forma correcta: "no sólo basado en percepciones".

Otros temas: Algunos temas sólo obtuvieron un comentario, por lo que no pudieron ser contrastadas, para estos casos se limitó a sintetizarlos en la siguiente lista:

a. Incluir la perspectiva de género en el análisis de necesidades socioeducativas.

b. Analizar las posibilidades de cada proyecto de intervención de manera colegiada en las primeras sesiones del programa

c. Al analizar el trato ético a los grupos vulnerables, fundamentarse en la ética consensuada

d. Un experto no entendió de que se trata "analizar las actitudes y aptitudes de un interventor exitoso"

e. Recalcar la importancia y atinencia del tema sobre presupuestos en el programa

f. Se debe adelantar el tema de la evaluación de la intervención antes de realizar el planteamiento de la misma

g. Hacer explícito que la autogestión y la transversalidad no son sólo temas, si no una forma de trabajar a lo largo de todo el curso

h. Es necesario reforzar el marco teórico del constructo empoderamiento

i. Hacer más explícito el bloque en el que se abordan las situaciones o estrategias para abordar, incursionar y trabajar con el grupo a intervenir, y recomendó hacer una simulación y, de ser posible, una práctica de intervención

\section{Conclusiones}

Con relación a los contenidos que debía contemplar un programa de formación para estudiantes universitarios para fungir como interventores en grupos vulnerables, se encontró que es necesaria la formación especializada para realizar intervenciones en grupos que perciban su entorno como inseguro y prepare a los interventores para enfrentar el contexto específico en el que se desempeñará el interventor (política, religión y tradiciones). Este contexto se debe tomar en cuenta al momento de diseñar las intervenciones junto con las condiciones de inseguridad y las limitantes de acción que implica la actividad delictiva en la región. Es importante señalar que, en cuanto a la Relación con la realidad, hubo muchas quejas de los grupos intervenidos, comentando que muchos proyectos no tenían ni pies ni cabeza o no se podían llevar a cabo por la misma situación de inseguridad percibida en la comunidad; los interventores también hicieron notar que al momento de implementar sus I.S. se encontraban con una dificultad extra a las previstas en su programa: el desconocimiento del entorno en el que realizarían sus actividades, pudiendo generar sentimientos de incompetencia e insuficiencia, independientemente de su nivel de experticia en sus respectivos campos de estudio.

Prospectiva. Revista de Trabajo Social e intervención social • No. $29 \bullet$ ene.-jun. $2020 \bullet$ pp. 131-150 e-ISSN: 2389-993X • Doi: 10.25100/prts.v0i29.7938

Universidad del Valle - Cali, Colombia 
Durante el trabajo colaborativo y el proceso de validación, se resaltó la importancia de cuidar el manejo del concepto vulnerabilidad para no adjudicarlo como algo inherente o natural en un grupo; no debe verse a la población a intervenir desde su vulnerabilidad, si no desde sus posibilidades de desarrollo.

Sobre los valores y actitudes que debe desarrollar un programa de formación para estudiantes universitarios como interventores en grupos vulnerables, la propuesta se basa en el trabajo colaborativo, tanto en la formación de interventores como en el diseño, aplicación y evaluación de las intervenciones a realizar, buscando la integración de la población objetivo a actividades tradicionalmente pensadas para coordinadores o interventores de los programas. Se resalta la importancia de que el interventor desarrolle un alto nivel de empatía y apertura al diálogo, además de que sea formado en el conocimiento y respeto del entorno y tradiciones de las comunidades a intervenir, para que su meta de desarrollo propuesta sea compatible con dicha comunidad. El discurso sobre el respeto a los grupos a intervenir como personas con su propia escala de valores y cosmogonía se repite en varias entrevistas, en los tres niveles de experticia, como una crítica a la forma hegemónica en la que suele producirse el conocimiento. Como lo señaló Caggiano (2012), los sistemas de categorías como las clasificaciones sociales son herramientas de poder que tienen consecuencias en la vida de las personas, por ello el interventor debe ser consciente de que su estilo de vida no es el deseable para todo el mundo, que sus valores no son absolutos y debe poder apreciar qué aprendizajes puede obtener de sus participantes para enriquecerse como interventor y como ser humano.

Se encontró la necesidad de ahondar y abogar por la necesidad del uso de indicadores, matrices FODA (Fortalezas, Oportunidades, Debilidades y Amenazas) y presupuestos en las intervenciones, debido a que se encontró una falta del uso de estas herramientas en los expertos en el diseño e implementación de intervenciones, aunque según algunas fuentes de la literatura consultada estas herramientas pueden respaldar el trabajo de campo, ayudar a pedir recursos para las actividades que se realizan y asegurar donatarios para proyectos futuros, al poder dar muestras de los avances o logro de objetivos. Estos temas son vistos aún como algo ajeno a las intervenciones, al menos, por la mayoría de los participantes de este estudio. Es importante recalcar el hecho de que los grupos autosustentables no deben considerarse una solución a un abandono del Estado como procurador de bienestar para el pueblo; en dichos grupos, como cualquier agrupación verdaderamente civil, debe hacerse conciencia sobre la necesidad de seguirle exigiendo al Estado que cumpla con ese rol.

Finalmente, se recomienda realizar un estudio donde se aplique este programa de formación, se contrasten los resultados con un grupo de control y se pueda realizar al menos una intervención piloto por los participantes. 


\section{Referencias bibliográficas}

Aguado, A. L., y Alcedo, M. A. (1999). La Escala de Valoración de Términos Asociados con Discapacidad en una muestra de EGB. Análisis y Modificación de Conducta, 25(103), 783806.

Aguado, D. A., Flórez, M. A., y Alcedo, M. A. (2004). Programas de cambio de actitudes ante la discapacidad. Psicothema, 16(4), 667-673.

ANUIES. (2016). Estado actual de las tecnologías de la información y las comunicaciones en las instituciones de educación superior en México. Estudio Ejecutivo. México: ANUIES.

Arenas, M. M. (2013). Un acercamiento sociológico a las formas de acción e intervención social. Acción social y acción social proyectada. Papeles del CEIC, 2(97), 1-33.

Arvon, H. (1982). La Autogestión. México: FCE.

Byrd, E. K., \& Elliott, T. R. (1988). Media and Disability: A Discussion of Research. En H. E. Yuker (Ed.), Attitudes toward Persons with Disabilities (pp. 82-95). New York: Springer.

Caggiano, S. (2012). El sentido común visual: disputas en torno a género, raza y clase en imágenes de circulación pública. Buenos Aires: Niño y Dávila Editores.

Castel, R. (2004). La inseguridad social. ¿Qué es estar protegido? Buenos Aires: Manantial.

Castells, M., y Borja, J. (2002). Local y global; la gestión de las ciudades en la era de la información. México: Taurus.

Charkiewicz, E. (2005). Corporations, the UN and Neo-liberal Bio-politics. Development, 48(1), 75-83.

Dahl-Ostergard, T., Moore, D., Ramírez, V., Wenner, M., y Bonde, A. (2003). Desarrollo rural de autogestión comunitaria ¿Qué hemos aprendido? Washington: Banco Interamericano de Desarrollo.

Duhau, E. (2002). Políticas sociales, ciudadanía y descentralización. En M. Calderón, W. Assies y T. Salman (Eds.), Ciudadanía, cultura política y reforma del Estado en América Latina (pp. 253-280). Morelia, México: El Colegio de Michoacán. Instituto Federal Electoral.

Donaldson, J. (1987). Cambio de actitudes hacia las personas deficientes. Siglo Cero, 112, 3038.

Elliott, T. R., \& Byrd, E.K. (1982). Media and Disability. Rehabilitation Literature, 43(11-12), 348-355.

Enríquez, I. (2011). Las concepciones sobre el desarrollo regional en las políticas públicas del sur-sureste mexicano y en los proyectos autogestivos de las comunidades locales: una contrastación a la luz de las inconsistencias del Plan Puebla-Panamá. OBETS. Revista de Ciencias Sociales, 6(2), 185-218.

Ezcurra, A. M. (1998). ¿Qué es el Neoliberalismo?: evolución y límites de un modelo excluyente. Buenos Aires: IDEAS.

Friedmann, J. (2001). Planificación en el ámbito público. Madrid: INAP.

Gómez, J., Latorre, A., Sánchez, M., y Flecha, R. (2006). Metodología comunicativa crítica. Barcelona: El Roure.

Prospectiva. Revista de Trabajo Social e intervención social • No. $29 \bullet$ ene.-jun. $2020 \bullet$ pp. 131-150 e-ISSN: 2389-993X • Doi: 10.25100/prts.v0i29.7938

Universidad del Valle - Cali, Colombia 
Cohetero-Hernández y González-Quiñones

Gómez, M. (2013). ¿Neoliberalismo autogestivo? La Cultura de Autogestión para el Desarrollo como una herramienta analítica. Para un análisis crítico del concepto de poscolonialidad. Contextualizaciones Latinoamericanas, (9), 1-12.

Habermas, J. (1987). Teoría de la acción comunicativa I. Racionalidad de la acción y racionalización social. Madrid: Taurus.

Habermas, J. (1997). Teoría de la acción comunicativa: complementos y estudios previos. Madrid: Cátedra.

Hale, C.R. (2002). Does multiculturalism menace? Governance, cultural rights and the politics of identity in Guatemala. Journal of Latin American Studies, 34(3), 485-524.

Harvey, D. (2005). Breve historia del Neoliberlismo. Madrid: Ediciones Akal.

Herrera, M., y Castón, P. (2003). Las políticas sociales en las sociedades complejas. Madrid: Ariel.

Idáñez, M., Ander-Egg, E., y Richmond, M.E. (2001). Diagnóstico social: conceptos y metodología. Buenos Aires, Argentina: Lumen.

Instituto Nacional de Estadística y Geografía. (2016). Encuesta nacional de seguridad pública urbana. Boletín de prensa, (12-17).

Instituto Nacional de Estadística y Geografía, y Secretaría de Gobernación. (2014). Encuesta de Cohesión Social para la Prevención de la Violencia y la Delincuencia (ECOPRED). Recuperado de https://www.inegi.org.mx/programas/ecopred/2014/.

Laval, C., y Dardot, P. (2017). La pesadilla que no acaba nunca: el neoliberalismo contra la democracia. España: Editorial GEDISA.

Lee, A. (2015). Intervención educativa: lo que necesita saber. Understood. Recuperado de https:/ / www.understood.org/es-mx/learning-attention-issues/treatmentsapproaches/educational-strategies/instructional-intervention-what-you-need-toknow.

Matzumura, J., Ayala, R., Aguilar, G., y Gutiérrez, H. (2008). El diagnóstico situacional en salud materno-infantil: Guía metodológica. Revista Peruana de Obstetricia y Enfermería, $4(1), 56-68$.

Mendizábal, A., y Errasti, A. (marzo, 2008). Premisas teóricas de la autogestión. En XI Jornadas de Economía Crítica. Asociación de Economía Crítica, Bilbao, España.

Mintz, F. (2006). Autogestión y anarcosindicalismo en la España revolucionaria. España: Traficantes de sueños.

Montero, I. (2014). Principios básicos de actuación en la animación sociocultural desde el ámbito de las personas mayores. Revista Interamericana de Educación de Adultos, 36(1), 92-103.

Mori, M. (2008). Una propuesta metodológica para la intervención comunitaria. Liberabit, 14(14), 81-90.

Ortiz, M. G. (2010). Neoliberalismo de Sur a Norte en América Latina, la Cultura de la Autogestión para el Desarrollo en Poblaciones Indígenas, los casos de Chile y México (tesis de doctorado). El Colegio de Michoacán. Zamora, México.

Paley, J. (septiembre, 2001). La "participación" y la "sociedad civil" en Chile: Discursos internacionales, estrategias gubernamentales, y respuestas organizacionales. En Congreso

Prospectiva. Revista de Trabajo Social e intervención social • No. $29 \bullet$ ene.-jun. $2020 \bullet$ pp. 131-150 e-ISSN: 2389-993X • Doi: 10.25100/prts.v0i29.7938

Universidad del Valle - Cali, Colombia 
Cohetero-Hernández y González-Quiñones

Latin American Studies Association (LASA 2001), Washington, Estados Unidos. Recuperado de http:/ / lasa.international.pitt.edu/Lasa2001/PaleyJulia.pdf.

Ramos, C. (2000). Enfoque comunitario, modernidad y posmodernidad: el trabajo social con la comunidad en tiempos de la globalización. Alternativas. Cuadernos de Trabajo Social, $8,185-204$.

Remedí, E. (marzo, 2004). La intervención educativa. En Reunión Nacional de Coordinadores de la Licenciatura en Intervención Educativa de la Universidad Pedagógica Nacional. México, D.F. México. Recuperado de https:// es.scribd.com/document/246065928/Conferencia-Eduardo-Remedi-LaIntervencion-Educativa.

Rodríguez, V. T. (1994). De los movimientos sociales a las metodologías participativas. En J. Delgado y J. Gutiérrez (Coords.), Métodos y técnicas cualitativas de investigación en ciencias sociales (pp. 399-426). Madrid: Síntesis.

Rodríguez, G. G., y Torres, G. (1994). Los agroproductores frente a las políticas neoliberales: El Barzón y COMAGRO. Espiral, 1(1), 129-176.

Rosanvallon, P. (1979). La Autogestión. Madrid: Editorial Fundamentos.

Salas, L. (2009). Migración y Feminización de la Población rural 2000-2005. El caso de Atitanac y La Encarnación, Villanueva, Zac (tesis de doctorado). Universidad Autónoma de Zacatecas Francisco García Salinas, Zacatecas, México.

Supiot, A. (Dir.). (1999). Au-delà de l'emploi. Transformations du travail et devenir du droit en Europe. París: Flammarion.

Touriñán, L. J. (2011). Intervención educativa, intervención pedagógica y educación: la mirada pedagógica. Revista portuguesa de pedagogía, 2, 283-307.

Verdugo, M. A., Jenaro, C., y Arias, B. (1995). Actitudes sociales y profesionales hacia las personas con discapacidad: Estrategias de evaluación e intervención. En M. A. Verdugo (Dir.), Personas con discapacidad: Perspectivas psicopedagógicas y rehabilitadoras (pp. 79-144). Madrid: Siglo XXI Editores.

Wright, B. A. (1984). Developing Constructive Views of Life with a Disability. En D. W. Krueger (Ed.), Rehabilitation Psychology: A Comprehensive Textbook (pp. 99-109). Rockville: Aspen Systems Corporation.

Yuker, H. E. (Ed.). (1988). Attitudes toward Persons with Disabilities. New York: Springer. 


\section{OTROS ARTÍCULOS DE PROSPECTIVA No. 29 DE 2020}

EDITORIAL

La Reconceptualización: una opción a la encrucijada del Trabajo Social en Colombia Víctor Mario Estrada-Ospina

\section{ARTÍCULOS}

Formación profesional y la práctica supervisada en el Servicio Social brasileño

Reginaldo Ghiraldelli

El rol docente del tutor y supervisor de prácticas de Trabajo Social: construcción de la reflexividad y el compromiso durante la formación a través de la supervisión

Carmina Puig-Cruells

Problematizando las prácticas preprofesionales en Trabajo Social. Desafios y perspectivas

Ruth Noemí Parola

Trabajo Social en contextos de formación política: la práctica transformadora

Angélica López-Yepes

Jackeline Cuenca-Echeverry

Investigación cuantitativa en una práctica universitaria de intervención en comunidad en el norte de México

Adán Cano-Aguilar
Interventores y comunidades vulnerables: una propuesta de formación para universitarios

Dulce Nayeli Cohetero-Hernández

Fidel González-Quiñones

Reagrupación familiar de menores en Aragón. Propuestas de acompañamiento en el proceso migratorio

Chabier Gimeno-Monterde

José David Gutiérrez-Sánchez

Aportaciones de la Educación en Derechos Humanos (EDH) y la Investigación Acción Participativa (IAP) en contextos de trabajo comunitario

Carla Cubillos-Vega

Condiciones para una NeoReconceptualización del Trabajo Social en Chile, Latinoamérica y el Caribe

Luis Vivero-Arriagada

Justicia sin daño: una apuesta por el fortalecimiento del acceso a la justicia Diego Fernando Acosta-Daza

\section{IN MEMORIAM}

Camilo Bautista Ochoa, un santandereano que se enamoró del Pacífico

Jesús Glay Mejía-Naranjo

\section{PROSPECTIVA}

Revista de Trabajo Social e Intervención Social 